\title{
On the measurement of the proton-air cross section using longitudinal shower profiles
}

\author{
R. Ulrich ${ }^{\mathrm{a}}$, J. Blümer ${ }^{\mathrm{a}}$, R. Engel ${ }^{\mathrm{a}}$, F. Schüssler ${ }^{\mathrm{a}}$, M. Unger ${ }^{\mathrm{a}}$ \\ ${ }^{a}$ Forschungszentrum Karlsruhe, Institut für Kernphysik, Germany
}

In this paper, we will discuss the prospects of deducing the proton-air cross section from fluorescence telescope measurements of extensive air showers. As it is not possible to observe the point of first interaction $X_{1}$ directly, other observables closely linked to $X_{1}$ must be inferred from the longitudinal profiles. This introduces a dependence on the models used to describe the shower development. The most straightforward candidate for a good correlation to $X_{1}$ is the depth of shower maximum $X_{\max }$. We will discuss the sensitivity of an $X_{\max }$-based analysis on $\sigma_{\mathrm{p}-\text { air }}$ and quantify the systematic uncertainties arising from the model dependence, parameters of the reconstruction method itself and a possible non-proton contamination of the selected shower sample.

\section{Introduction}

One of the most important parameter needed for interpretation of air shower measurements is the proton-air cross section $\sigma_{\mathrm{p}-\text { air }}$, which is not well known at cosmic ray energies. Collider measurements of the proton-(anti)proton cross section have to be extrapolated over a wide energy range to be used in models of nuclear effects to predict the proton-air cross section at ultra high energies. On the other hand, it might be possible to measure $\sigma_{\mathrm{p}-\text { air }}$ by analyzing extensive air showers (EAS).

The point of first interaction in slant depth $X_{1}$ follows the exponential distribution

$\frac{d P\left(X_{1}\right)}{d X_{1}}=\frac{1}{\lambda_{\mathrm{p}-\text { air }}} \cdot e^{-X_{1} / \lambda_{\mathrm{p}-\mathrm{air}}}$.

The cross section $\sigma_{\mathrm{p}-\text { air }}$ is related to the absorption length $\lambda_{\mathrm{p}-\text { air }}$ as $\lambda_{\mathrm{p}-\text { air }}=M_{\text {mean }} / \sigma_{\mathrm{p} \text {-air }}$, with $M_{\text {mean }}$ being the mean mass of a target (air) particle.

The depth and characteristics of the first interaction of the primary cosmic ray particle with a molecule of the atmosphere determines to a large extent the resulting shower shape. This implies strong correlations of the first interaction with experimentally observable parameters of the shower.

Several air shower experiments have provided measured values of $\sigma_{\mathrm{p}-\text { air }}$ by exploiting correla- tions of $X_{1}$ with observables found by using air shower simulations [1/2]3/45]. Of course, these results depend strongly on the used simulations and especially on the chosen high energy hadronic interaction model [6]7.

The HiRes collaboration proposed a new technique which appeared to be almost independent of the hadronic interaction model $[8$. The idea is to decompose the measured distribution of shower maxima $d P\left(X_{\max }\right) / d X_{\max }$ into the two independent distributions of $X_{1}$ and $\Delta X$ with

$X_{\max }=X_{1}+\Delta X$.

Using the introduced $\Delta X$ one can express the $X_{\max }$ distribution as folding integral

$P\left(X_{\max }\right)=\int_{0}^{\infty} d X_{1} P\left(X_{1}\right) \cdot P\left(\Delta X \mid X_{1}\right)$

of the $X_{1}$ and $\Delta X$ distributions, whereas in the HiRes [8] approach $P\left(\Delta X \mid X_{1}\right)=P(\Delta X)$ is used.

Measuring $\sigma_{\mathrm{p}-\text { air }}$ means to deconvolve the $X_{\max }$ distribution using a known $\Delta X$ distribution.

The distribution of $\Delta X$ needs to be inferred from simulations.

\section{Simulation study}

To investigate the deconvolution technique, we simulated sets of proton-induced showers of $10 \mathrm{EeV}$ using CONEX [9] with the high energy 

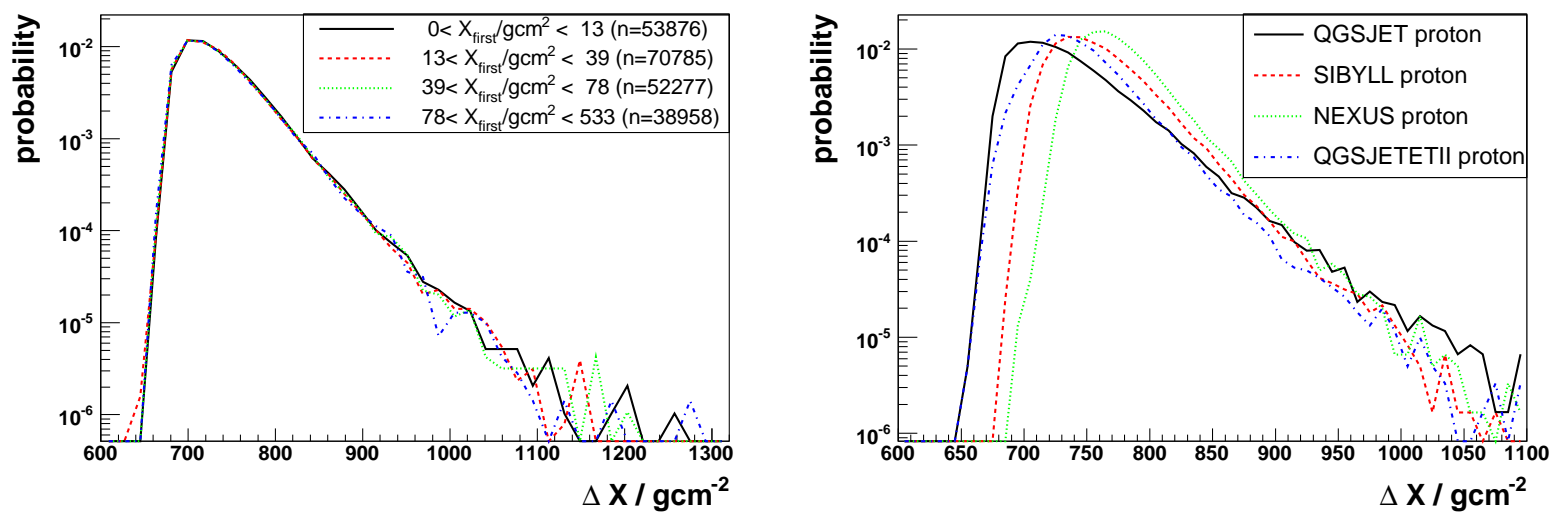

Figure 1. Left panel: Dependence of the $\Delta X$-distribution on $X_{1}$. Right panel: Interaction model dependence of $\Delta X$-distribution.

interaction models QGSJET01 [10], SIBYLL2.1 [11, NEXUS3 [12, and QGSJETII.3 [13. In addition helium, CNO and gamma-ray primaries were simulated with QGSJET01. Each set of simulations contains 200.000 events.

\section{Universality of $\Delta X$ distribution}

One of the important conditions for the applicability of the method proposed in [8] is the $X_{1^{-}}$ independence of the $\Delta X$ distribution. For a given model, this is indeed the case as shown in Fig. 1] (left). The universality in $X_{1}$ has been verified for proton primaries using NEXUS3, SIBYLL2.1, QGSJETII.3, and QGSJET01 at $1 \mathrm{EeV}$ and $10 \mathrm{EeV}$.

Therefore one can use

$P\left(\Delta X \mid X_{1}\right)=P(\Delta X)$.

However, the resulting $\Delta X$ distribution depends strongly on the hadronic interaction model used for shower simulation (Fig. 11, right). The maximum of the $\Delta X$ distribution is shifted by up to $60 \mathrm{~g} / \mathrm{cm}^{2}$ and the slope beyond the maximum changes by up to a factor of 1.36. This introduces a principal model dependence to the deconvolution technique. It can be tried to reduce this dependence by adding an additional degree of freedom, $X_{\text {shift }}$, which allows the $\Delta X$ distribution to shift along the $\mathrm{x}$ axis

$P(\Delta X) \rightarrow P\left(\Delta X+X_{\text {shift }}\right)$.

It is much more difficult to reduce the modeldependence of the shape of the $\Delta X$ distribution. This will not be discussed in this paper but we shall show the impact of this model-dependence on the resulting $\sigma_{\mathrm{p}-\text { air }}$ reconstruction.

\section{Study of the deconvolution method}

For each model, a Monte Carlo data set of 50.000 events was generated and analyzed with the $\Delta X$ distributions found previously. No detector effects are considered. Two free parameters $\left(\lambda_{\mathrm{p}-\text { air }}\right.$ and $\left.X_{\text {shift }}\right)$ are fitted using $\chi^{2}$ minimization and

$$
\begin{aligned}
\frac{d P\left(X_{\max }\right)}{d X_{\max }} & =\int_{X_{1, \min }}^{X_{1, \max }} d X_{1} \frac{1}{\lambda_{\mathrm{p}-\text { air }}} e^{-X_{1} / \lambda_{\mathrm{p}-\text { air }}} \\
& \times\left(\frac{d P\left(\Delta X+X_{\text {shift }}\right)}{d \Delta X}\right)_{\text {model }} \cdot(6)
\end{aligned}
$$

The fit results are shown in Tab. 1 for all combinations of hadronic interaction models and $\Delta X$ distributions. The diagonal elements of the table represent matching models for data and $\Delta X$. In these cases the true cross section of the input model can be retrieved very well by the convolution method. It is interesting that for models with a similar shape of the $\Delta X$ distribution 
Table 1

Analysis results of $\sigma_{\mathrm{p}-\text { air }}$ in mb for $10 \mathrm{EeV}$ proton showers. The results for $X_{\text {shift }}$ are given in $\mathrm{g} / \mathrm{cm}^{-2}$. The expected cross sections are $532 \mathrm{mb}$ (QGSJET01), $612 \mathrm{mb}$ (SIBYLL 2.1), $542 \mathrm{mb}$ (NEXUS), and $562 \mathrm{mb}$ (QGSJETII).

\begin{tabular}{|c|c|c|c|c|c|c|c|c|}
\hline \multirow[b]{3}{*}{ data model } & \multicolumn{8}{|c|}{$\Delta \mathrm{X}$ model used for reconstruction } \\
\hline & \multicolumn{2}{|c|}{ QGSJET } & \multicolumn{2}{|c|}{ SIBYLL } & \multicolumn{2}{|c|}{ NEXUS } & \multicolumn{2}{|c|}{ QGSJETII } \\
\hline & $\sigma_{\mathrm{rec}}$ & $X_{\text {shift }}$ & $\sigma_{\mathrm{rec}}$ & $X_{\text {shift }}$ & $\sigma_{\mathrm{rec}}$ & $X_{\text {shift }}$ & $\sigma_{\mathrm{rec}}$ & $X_{\text {shift }}$ \\
\hline QGSJET01 & $537.1 \pm 5.5$ & $1.7 \pm 0.8$ & $465.3 \pm 4.1$ & $-29.8 \pm 0.8$ & $447.9 \pm 3.2$ & $-46.9 \pm 0.8$ & $467.6 \pm 3.7$ & $-19.6 \pm 0.7$ \\
\hline SIBYLL & $802.8 \pm 12$ & $36.1 \pm 0.7$ & $609.2 \pm 5.7$ & $0.7 \pm 0.5$ & $572.8 \pm 4.5$ & $-17.4 \pm 0.5$ & $613.3 \pm 5.7$ & $10.6 \pm 0.5$ \\
\hline NEXUS & $749.9 \pm 10$ & $55.1 \pm 1.2$ & $569.1 \pm 3.9$ & $19.5 \pm 0.9$ & $543.1 \pm 4.0$ & $1.5 \pm 0.5$ & $576.5 \pm 4.6$ & $29.5 \pm 0.8$ \\
\hline QGSJETII & $697.1 \pm 8.2$ & $27.0 \pm 0.8$ & $553.2 \pm 4.3$ & $-7.5 \pm 0.6$ & $521.6 \pm 3.7$ & $-26.1 \pm 0.3$ & $562.3 \pm 4.4$ & $2.1 \pm 0.4$ \\
\hline
\end{tabular}

(QGSJETII and SIBYLL) the reconstruction still works rather well, whereas for combinations of models which have a very different shape of $\Delta X$ (QGSJET and NEXUS) the results differ up to $200 \mathrm{mb}$

\subsection{Importance of fit range}

The reconstructed value of $\sigma_{\mathrm{p}-\text { air }}$ is very sensitive to the chosen starting point of the fit range, as shown in Fig. 2 (left). The reason for this is the introduced $X_{\text {shift }}$ parameter which needs the peak of the $X_{\max }$ distribution to be restricted. To fit only the slope after the peak of the $X_{\max }$ distribution is not sufficient if $X_{\text {shift }}$ is treated as free parameter.

Restricting the end point of the fitting range mainly increases the statistical uncertainty of the fit result. The true value of $\sigma_{\mathrm{p}-\text { air }}^{\text {true }}$ is still within the statistical errors of the reconstructed values (see Fig. 2, right).

\subsection{Impact of other elements}

If the selected showers do contain a fraction of light elements other than protons, the shape of the measured $X_{\max }$ distribution will be altered. A possible contribution of helium will mostly distort the region of the peak of the $X_{\max }$ distribution. Since the peak of the $X_{\max }$ distribution is important for the fit itself, the reconstruction of $\sigma_{\mathrm{p}-\text { air }}$ is affected (Fig. 3, top)

Contamination with CNO showers mainly influences the rising edge of the $X_{\max }$ distribution. The impact on the fit result can be limited by shifting the starting point of the fit range more towards the peak of the distribution. Using a short fit range, the effect on the reconstruction of $\sigma_{\mathrm{p}-\text { air }}$ can be neglected for small fractions of CNO (Fig. 3, middle).

\subsection{Impact of gamma-ray primaries}

Gamma-ray primaries produce an $X_{\max }$ distribution which is shifted to higher $X_{\max }$ by about $200 \mathrm{~g} / \mathrm{cm}^{2}$ with respect to protons. Therefore the influence of gamma-ray showers can be reduced by setting the end of the fitting range appropriately (Fig. 3, bottom). Nevertheless already a small contribution of gamma-rays does affect the $X_{\max }$ distribution significantly because of the low number of proton events at high $X_{\max }$. It should be noted that even if cosmic ray sources would accelerate only protons, a small fraction of gammarays is expected due to the GZK energy loss process [14].

\section{Summary}

The folding integral method would be very powerful given the true $\Delta X$ distribution is known. Input cross sections can be reconstructed within statistical uncertainties of a few mb. Unfortunately, the $\Delta X$ distribution does depend on the high energy hadronic interaction model used during air shower simulation. The $\Delta X$ model dependence can be reduced by adding a new degree of freedom $X_{\text {shift }}$ to the fit but it still amounts up to $\sim 100 \mathrm{mb}$. On the other hand, treating $X_{\text {shift }}$ as free parameter increases the sensitivity of $\sigma_{\mathrm{p}-\text { air }}$ to a possible helium contamination of the $X_{\max }$ distribution. Medium mass cosmic ray primaries have only limited influence on the reconstructed $\sigma_{\mathrm{p} \text {-air. }}$. Even a small fraction of gamma-ray primaries has a significant impact on the reconstruction results.

To use the folding integral method for a reliable cross section measurement, the model dependence of the $\Delta X$ distribution has to be reduced significantly. 

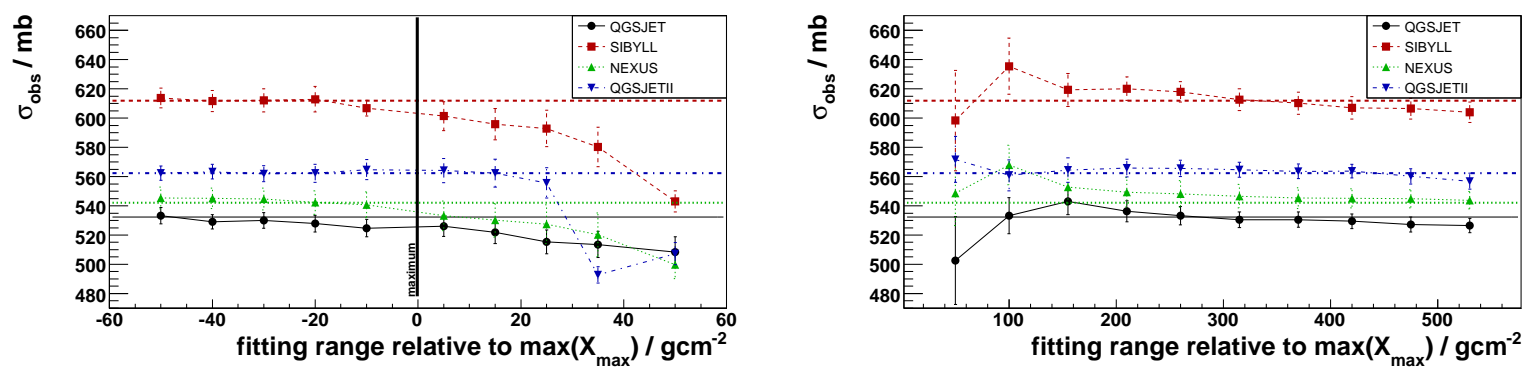

Figure 2. Impact of the begin (left panel) and the end (right panel) of the fit range of the $X_{\max }$ distribution (pure proton composition at $10 \mathrm{EeV}$ ). On the $\mathrm{x}$ axis the distance to the peak of the $X_{\max }$ distribution is shown. The horizontal lines denote the true value of $\sigma_{\mathrm{p}-\text { air }}^{\text {true, model }}$ for each interaction model.

\section{REFERENCES}

1. M. Honda et al., Phys. Rev. Lett. 70 (1993) 525

2. T. Hara et al., Phys. Rev. Lett. 50 (1983) 2058

3. R. M. Baltrusaitis et al., Phys. Rev. Lett. 52 (1984) 1380

4. M. Aglietta et al. Nucl. Phys. A (Proc.Suppl.) 75A (1999) 222

5. S. P. Knurenko et al., 26th ICRC Utah 1 (1999) 372

6. T. K. Gaisser et al., Phys. Rev. D 36 (1987) 1350

7. R. Engel et al., Phys. Rev. D 58 (1998) 014019

8. K. Belov et al., Nucl. Phys. (Proc.Suppl.) 151 (2006) 197

9. T. Bergmann et al., Astropart. Phys. 26 (2007) 420

10. N.N. Kalmykov et al., Nucl. Phys. B (Proc. Suppl.) 52B (1997) 17

11. R. Engel et al., Proc. 26th ICRC Utah 1 (1999) 415

12. K. Werner et al., Heavy Ion Phys. 21 (2004) 279

13. S. Ostapchenko, Nucl. Phys. (Proc.Suppl.) 151 (2006) 143

14. G. Gelmini et al., astro-ph/0506128
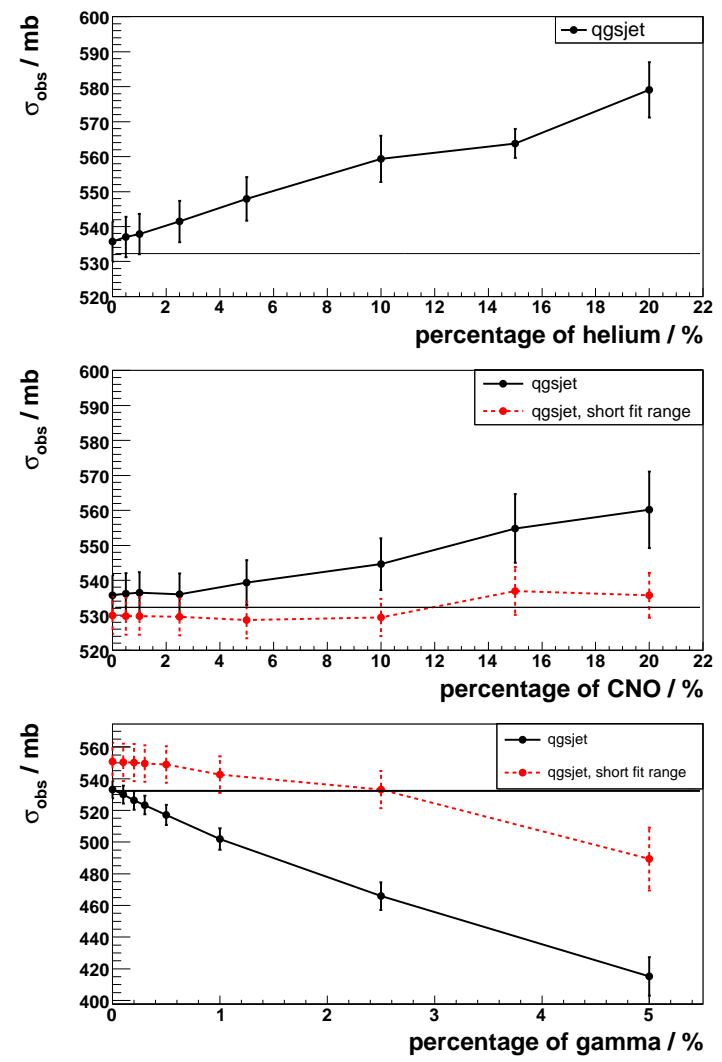

Figure 3. Impact of non-proton contributions in the analyzed shower sets on the reconstruction of $\sigma_{\mathrm{p}-\text { air }}$. The horizontal lines denote the true value of $\sigma_{\mathrm{p} \text {-air }}^{\text {true, }}$ of the used high energy interaction model. 